\title{
XXXI. Die Debye-Scherrer-Methode zur Untersuchung von Kristallstrukturen.
}

\author{
Von \\ O. Pauli in Frankfurt a. $M$. \\ (Mil 11 Figuren im Toxt und einer Tafel.)
}

Die Entdeckung Laues, daß Rüntgenlicht durch Kristallgitter gebeugt wird und auf einer photographischen Platte ein Muster von Flecken ergibl, aus dem man die Symmetrie der Kristalle erkennen, den Gittertyp bestimmen und die Gitterkonstante berechnen kann, eröfnete dem Kristallographen ein neues, weites Forschungsfeld und führte bereits zu einer Reihe schöner Resultate.

Das Laue-Verfahren wurde durch die Braggs wesentlich vereinfacht und für die Bestimmung von Kristallstrukturen brauchbar gemacht. Die Braggs wiesen nach, daß man den Laue-Effekt entstanden denken kann durch Reflexion monochromatischer Röntgenstrahlen an den Gilterebenen der Kristalle. Sie benutzten für ihre Versuche monochromatisches Röntgenlicht und zeigten, da $\beta$ dieses nur dann an einer orientiert aufgestellten Kristallfläche zur Reflektion gelangen kann, wenn

$$
n \cdot \hat{\lambda}=2 d \cdot \cos \varphi
$$

ist. Hierin bedeutet $n$ die Ordnungszahl der Reflexion, $\lambda$ die Wellenlänge des Röntgenlichts, $d$ den Abstand zwischen zwei benachbarten Gitterebenen und $p$ den optischen Reflexionswinkel. Diese Methode setzt also ebenfalls orientierte Kristallflächen voraus.

Eine weitere Vereinfachung der Laue-Methode bedeutet das Verfahren von Debye-Scherrer. Es setzt weder orientierte Kristalfflächen noch die Kenntnis des Kristallsystems der zu untersuchenden Substanz voraus. Es benutzt wirr durcheinander liegendes Kristallpulver und bringt an diesem monochromatische Röntgenstrahlen zur Reflexion. Es kommen bei ihm gleichzeitig alle Gilterebenen des Kristalls, die merklich reflektieren, zur 
Geltung. Das Kristallpulver wird in cinem Papierröhrchen in die Achse eines Filmzylinders gebracht und dann mit einem schmalen Bündel parallelen, monochromatischen Röntgenlichles durchstrahlt. Die reflektierten Strahlen bilden einen Kreiskcgelmantel und erzeugen auf dem Film Linien, aus deren Lage zueinander oder zum Durchstichspunkt des Primärstrahls die Reflexionswinkel berechnet werden können.

Die übrigen für die Erforschung von Kristallstrukturen benutzten Verfahren schließen sich mebr oder weniger an diese drei Verfahren an. Es ist wohl augenscheinlich, daß das Debye-Scherrer-Verfabren große Vorteile gegenüber den Methoden von Laue und der Braggs bietet. Diese Vorteile liegen vor allem darin, daß durch die Benutzung von Kristallpulver die Orientierung der zu untersuchenden Substanz wegfällt und daß alle merklich reflektierenden Gitlerebenen des Kristalls auf dem Film Interferenzlinien erzeugen. Ein einziger guter Film genügt also zur Auswertung. Da Kristallpulver benutzt wird, kann man nach dicser Methode auch die sogenannten amorphen Substanzen untersuchen. Diese Vorzüge sind cs wohl gewesen, wclche das Interesse der Fachgenossen dieser Methode in letzter Zeit vor allen zugelenkt haben.

\section{Die Apparatur.}

\section{1. a) Die Röhre für monochromatisches Röntgenlicht.}

Die Typen von Röntgenröhren, die zur Herstellung von Debyescherrer-Photogrammen vorwiegend benutzt werden, sind von Rausch von Traubenberg und von M. Siegbahn konstruiert. Da die letztere große Vorteile hinsichtlich der Intensität der Strahlung und der Auswechselung des Antikathodenmaterials bietet, soll hier nur auf diese eingegangen werden. Die vom Verfasser benutzte Röhre ist in Form und Größe der von Hadding ${ }^{1}$ ) beschriebenen nachgebildet. Der Metallgußkörper ist bei ilır durch zwei Metalldruckkörper ersetzt, die so ineinandergepaßt sind, daß zwischen ihnen noch ein Raum für das Kühlwasser bleibt. Das Palladiumröhrchen befindet sich nicht direkt an der Röhre, sondern ist an dem Verbindungsrohr zwischen Rühre und Luftpumpe angebracht. $\Lambda$ Is Isolator für die Kathode wurde zunächst der Isolator Nr. 0,8797 der Berliner Porzellanmanufaktur benutzt, der von Hadding ${ }^{1}$ ) angegeben ist. Der Isolator erfüllt seinen $Z$ weck vorzüglich. Die Kathode darf nur nicht zu stramm in ihn eingepaßt werden, da er sonst bei dem großen Wärmeausdehnungsvermögen des Aluminiums zersprengt werden kann. Zum Aufkitten dieses Isolators auf den Metallkörper der Röhre wurde Akkumula-

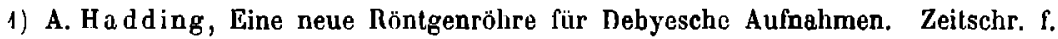
Phys. 1920, 3. Bd., 5. Heft. 
Lorenkitt benutzt. Da diese Isolatoren nur auf Bestellung angefertigt und auch nur in größerer Anzahl geliefert werden, wurde später als vollwertiger Ersatz ein Röhrenkopf benutzt, wie ihn Fig. 1 im Durchschnitt wiedergibt. $a$ ist ein Messingkreisring von $10 \mathrm{~mm}$ Dicke und von einem Durchmesser, der um 10-20 mm größer ist als der des Metallkörpers $c$ an der Auflrittstelle. Der Ring besitzt bei $b$ eine ringförmige Vertiefung, in welche der Metallkörper $c$ der Röhre paßt. In die mitllere Durchbohrung $d d$ der Kreisscheibe ist ein $50 \mathrm{~mm}$ langes Messingrohr $e$ eingelötel. An diesem und an der Kreisscheibe $a$ ist ein Kühlmantel $f$ mit den Ansatzröhren $g_{1}$ und $g_{2}$ befestigt. Durch das Rohr $e$ paßt das Isolierrohr $h$. Zur Isolation verwende man ein Porzellanrohr, welches in den Fabriken vorrïtig ist. Lis soll etwa $300 \mathrm{~mm}$ lang sein, eine Wandstärke von $10 \mathrm{~mm}$ und einen inneren Durchmesser von $3 \breve{-}-40 \mathrm{~mm}$ besilzen. Die Verwendung von zwei ineinanderpassenden Glasrohren zur Isolation ist weniger empfehlenswert, da sie leichter beim Erhitzen zerspringen und viel schlechter isolieren als Porzellan. In das Isolierrohr wird das Kühlrohr $i$ mit der Aluminiumkathode $k$ hineingeschoben und am Isolierrohr bei $l$ mit weißem Siegellack (ocler Akkumulatorenkitt) so festgekiltet, daß der lland der Kathode mit dem Ende des Isolierrohrs zusammenfälll. Kathode uncl Kühlrohr müssen bequem in das Isolierrohr passen, damit letzteres nicht bei Erwärmung der Kathode durch die Metallteile zersprengt wird. Das lsolierrohr wird dann mil der Kathode durch das Rohr $e$ geschoben und au diesem und dem Küblmantel $f$ mit weißem Siegellack (oder Akkumulatorenkitt) feslgekittet. Die Entfernung: zwischen Kathode und Antikathode ist bei den

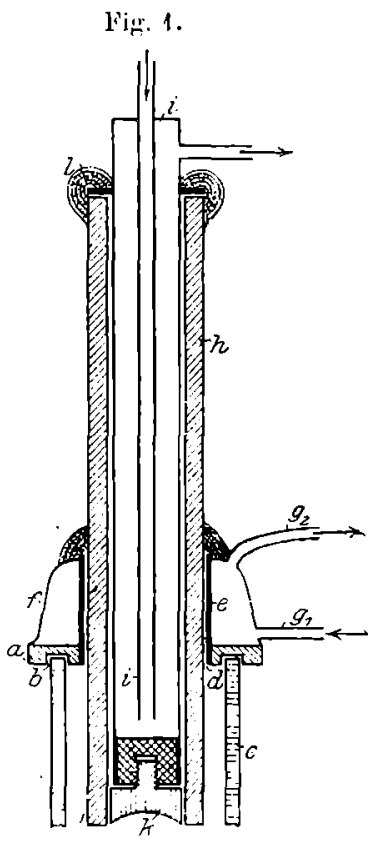
Siegbahnröhren etwa gleich dem vierfachen Betrage des Krümmungsradius der Kathode. In die Einsenkung $b$ des nun fertigen Röhrenkopfes wird der Metallkürper $c$ geschoben und beide Teile mit Pizein aneinander luftdicht befestigt.

Die drei Fenster an der Anlikalhodenrühre wurden mit Aluminiumfolie von $\frac{1}{20} \mathrm{~mm}$ Dicke überdeckt und mit Pizcin angekittet. Nach längerer Bcnutzung der Röhre wechsle man die Aluminiumfolie aus, damil sie nicht allzu dick mit zerstäubtem Antikathodenmetall überzogen wird.

Zum Evakuieren der Röhre wurde eine Gaede-Quecksilbertrommelpumpe benutzt, deren Vorvakuun vermittelst einer Wasserstrablluftpumpe 
hergestellt wurde. Diese Luftpumpenkombination arbeitet sehr gleichmäßig, läßt sich schnell regulieren und ist bequem in der Handhabung. Sie besitzt nur den einen Nachteil, daß sie die in der Metallröhre stets reichlich enthaltene Feuchtigkeit sehr langsam entfernt. Zwischẹn Röbre und Pumpe wurde ein Glasrohr beiderseits vermittels Schliffe eingepaßt. Es ist zweckmäßjg, in dessen Mitte eine Glasfederung einzuschmelzen, welche das Einsetzen der Schliffe erleichtert. Ferner ist es vorteilhaft, zwischen Glasfeder und Pumpe an dem Verbindungsrohr einen Abstellhahn anzubringen, einerseits um das Eindringen von Quecksilberdampf in die Röhre zu verhüten, wenn diese nicht benutzt oder wenn nicht gepumpt wird, andererseits stellte es sich heraus, daß die Röhre, wenn sie konstant geworden ist, viel ruhiger arbeitet, wenn der Weg zur Pumpe versperrt ist. Um zu verhüten, daß die Röhre zu hart wird, wurde $z$ wischen Röhre und Feder ein Schliff angeschmolzen, an welchem sich ein Palladiumrührchen (sogenannte Osmo-Regenerierung) befand. /wischen Feder und Röhre, möglichst in der Nähe der letzteren, wurde noch ein zweiter Schliff an dem Verbindungsrohr angesetzt und an diesen ein Glaskolben mit mehreren Litern Inhalt angebracht, in welchem sich etwas Phosphorpentoxyd befand. Dieser Kolben verkleinert durch sein Volum die Wirkung der Druckschwankungen in der Röhre, die öfters durch Gasabgabe aus dem Metallkörper entstehen. Das Phosphorpentoxyd im Kolben nimmt die etwa in der Röhre enthaltene Feuchtigkeit auf und beseitigt dadurch teilweise den Mangel der GaedeQuecksilbertrommelpumpe, die ja Feuchtigkeit nur sehr langsam abpumpt. Die Röbre ist etwa nach halbstündigem Pumpen gebrauchsfertig. Nach viertelstündigem Pumpen wurde gewöhnlich schon Strom durch die Röhre geschickt. Die dadurch erhitzten Metaliteile gaben dann schneller Luft und Feuchtigkeit ab. Während des Betriebes wurde die Konstanz der Föhre durch einen Zinksulfidschirm geprüft, welcher sich in einer Messingfassung befand, mit Bleiglas überdeckt war und an der Antilathodenröhre vor dem Aluminiumfenster angeschraubt wurde.

\section{1. b) Die Stromquelle.}

Als primärer Strom wurden 240 Volt Wechselstrom der städtischen Leitung benutzt. Dieser wurde durch eine Transformatorenlage von Siemens \& Halske, wie sie vielfach von Ärzten benutzt wird, herauftransformiert und durch einen Nadelschalter gleichgerichtet. Die Rühre wurde durchschnittlich bei $40-50000$ Volt mit 10-1: Milliampère belastet. Die Stromstärke wurde durch ein Milliampèremeter, die Spannung durch eine Parallelkugelfunkenstrecke von $25 \mathrm{~mm}$ Kugeldurchmesser gemessen. Das Optimum der Belastung kann man auf folgendem Wege finden: Man befesligt an dem einen Fenster der Antikathodenröhre den Zinksulfidschirm und an dem 
zweiten eine enge Spaltblende. Vor letztere stellt man einen Steinsalzwürfel unler dem Glanzwinkel auf und beobachtet die reflektierte Strahlung auf einem Fluoreszenzschirm. Man verändert nun die Belastung und die Höhe des Vakuums solange, bis die Kupfer- $\alpha$ - und $\beta$-Linien auf dem Fluoreszenzschirm mit maximaler Intensität erscheinen und prüft am Zinksulfidschirm, ob bei dieser Belastung die Robre ruhig arbeitet. Erhitzt sich auch bei längerem Betricbe der Röhre bei dieser Belastung der Antikathodenmantel nicht, so hält man am primären und sekundären Stromkreise die Belastung konstant und reguliert nun nur noch die Entladungsbedingungen durch Evakuieren resp. durch Erhitzen des Palladiumröhrchens.

\section{1. c) Die Kühlung der Röhre.}

Bei Benutzung des Isolators Nr. 0,8797 sind die Antikathode, der Rührenmantel und die Kathode zu kühlen, bei Benutzung des vorher beschriebenen Röhrenkopfes auch noch dieser. Antikathodenkühlung, Kühlung des Röhrenmantels und die des Röhrenkopfes wurden hintereinander geschaltet und mit der Wasserleitung verbunden. Da Antikathode, Rührenkörper und Röhrenkopf sehr stark gekühlt werden müssen, empfiehlt es sich, sie durch Druckschlauch zu verbinllen. Die Wasserkühlung der Kathode, für wolche ein langsamer Wasserstrom genügl, wurde, um Kurzschluß zu vermeiden, aus einem isoliert aufgehängten Auslaufgefäß gespcist. Auch der Ablauf des Kathodenküh/wassers war unabhängig von der anderen Kühlung. Bei guter Kühlung kann die Röhre über 2 Stunden ohne Unterbrechung benutzt werden.

\section{Die Kamera.}

Die Kameras, die zur Herstellung von Debye-Scherrer-Phologrammen benutzt werden, müssen folgende Bedingungen erfüllen: Sie müssen lichtdicht abschließen, sie müssen den Film stets und überall in konstanter Entfernung vou der Zylinderachse enthalten, sie müssen eine genaue Zentrierung des Pulverstäbchens, wenn müglich auch bei Tageslicht, zulassen, und sie dürfen endlich auch bei längerer Belichtung keine Reflexionserscheinungen auf dem Film hervorbringen. Gleichzeitig sollen sie bequem in der Handhabung sein und möglichst nahe an die Fenster der Röntgenröhre herangebracht wcrden künnen, tlamit die Strahlung durch die Blende nicht zu stark geschwächt wird. Da man ein Strahlenbündel mit endlicher Öffnung benutzt, nimmt die Intensitït der Strahlung angenähert quadratisch mit der Entfernung von der Röhre ab.

\section{2. a) Kamera $I$.}

Die meisten der eben genannten Bedingungen erfüllt eine Kamera, welche nacb Angabe der Herren Debye und Scherrer für die Aufnahmen hergestelit 
wurde (Fig. 2). Auf einem Metalltisch $a$ mit drei Stellschrauben ist ein $70 \mathrm{~mm}$ hoher Messingzylinder $b$ so befestigt, daß die Zylinderachse senkrecht zum Tisch steht. Der innere Radius des Zylinders beträgt $29 \mathrm{~mm}$.

Fig. 2.

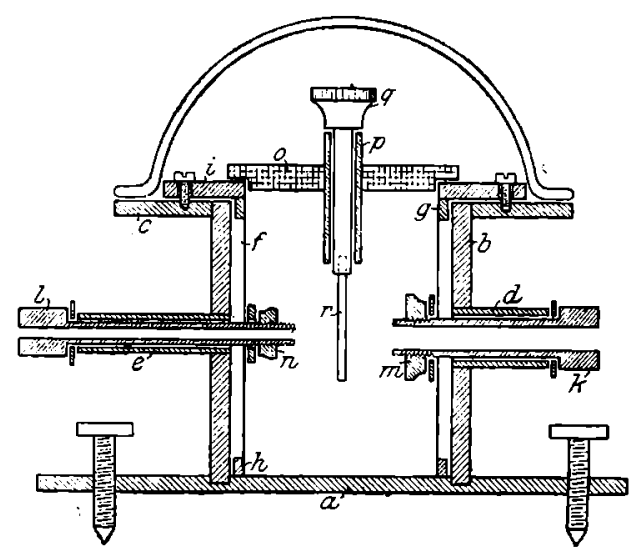

Das obere Ende des Zylinders schließt mit einem oben plangeschliffenen Metallring $c$ von $30 \mathrm{~mm}$ Breite ab, der am Zylinder angelötet ist. In mittlerer Höhe des Zylinders $b$ sind diametral zwei Ansatzröhren $d$ und $e$ befestigt, $d$ von einem inneren Durchmesser von $8 \mathrm{~mm}, e$ von einem inncren Durchmesser von $6 \mathrm{~mm}$. In den Zylinder $b$ paßt ein zweiter Zylinder $f$. Dieser besteht aus zwci Lagen von schwarzem Papier, ist cbenfalls $70 \mathrm{~mm}$ hoch und hat einen äußeren Durchmesser von $50 \mathrm{~mm}$.

An $f$ ist oben und unten je ein Metallring $g$ und $h$ von $4 \mathrm{~mm}$ Höhe und 2 $\mathrm{mm}$ Breite außen angeklebt. An $g$ ist oben ein Metallring $i$ von $10 \mathrm{~mm}$ Breite angelötet. Der Papierzylinder $f$ mit seiner Metallfassung $g$ und $h$ kann in den Zylinder $b$ hineingeschoben werden. Zur Dichtung legt man auf den Boden des Zylinders $b$ ène Kreisscheibe aus Samt und zwischen die Ringe $e$ und $i$ einen Ring von schwarzem Papier. Die beiden oberen Ringe $c$ und $i$ können durch Schrauben fest aneinandergeschraubt werden. Der Pappzylinder $f$ besilzt Durchbohrungen in gleicher Höhe und in gleichem Ausma $B$ wie der Zyylinder $b$, so daß man durch die Ansalzröbren $l$ und $e$ und durch die Durchbohrung des Papierzylinders Messingröhren bei $d$ von $8 \mathrm{~mm}$ äußerem und $6 \mathrm{~mm}$ innerem Durchmesser und bei $e$ von $6 \mathrm{~mm}$ äußerem und $4 \mathrm{~mm}$ innerem Durclimesser hindurchschieben kann. Diese Röhren haben an ihrem einen Ende Anschläge $l i$ und $l$ und an den anderen Gewinde mit Schraubenmuttern $m$ und $n$. 'Zur Dichtung legt man $z$ wischen die Muttern bei $m$ und $n$ und den Papierzylinder $f$ Gummiringe. Auf den oberen, am Pappzylinder befestigten Metallringen $g$ und $i$ wird eine Kreisscheibe $o$ aus Ilessing versenkt aufgesetzt, durch deren Mitte eine Messingröhre $p$ geht, die als Führung für eine Achse $q$ dient, die mit der Zylinderachse zusammenfällt. An dieser wird das Pulverstäbchen $r$ befestigt.

\section{2. b) Kamera II.}

Die andere zur Herstellung von Debye-Scherrer-Photogrammen vom Verfasser benutzte Kamera ist einfacher in der Handhabung und 
kleiner im Volum als Kamera I. Sie gewährt außerdem den Vorteil, daß das Stibchen noch genauer zentrisch zum Film liegt, als dies bei Kamera I der Fall ist. Auch liegt hier der Film genau kreisförmig um das Stäbchen. Der Fuß $a$ mit den Stellschrauben ist an dieser Kamera fortgelassen (Fig. 3 a). Der äußere Messingzylinder $b$ ist unten durch eine Messingplatte $s$ abgeschlossen und hat oben einen Metallring $c$ von $10 \mathrm{~mm}$ Breite. Der innere Zylinder $f_{1}$ besteht aus einem Messingrohr von $50 \mathrm{~mm}$ äußerem Durchmesser. Dic Dichtung am oberen $(g$ und $i)$ und unteren Ende (h) ist genau so wic bei Kamera I, ebenso die Durchbohrung. Die Ansatzröhren $d$ und $e$ und die Durchführungsrühren mit den Anschlägen $k$; und $l$ und mit den Muttern $m$ und $n$ der Kamera I (vgl. Fig. 2) sind hier durch zwei Ansatzröhren $t$ und $u$ von $6 \mathrm{~mm}$ resp.

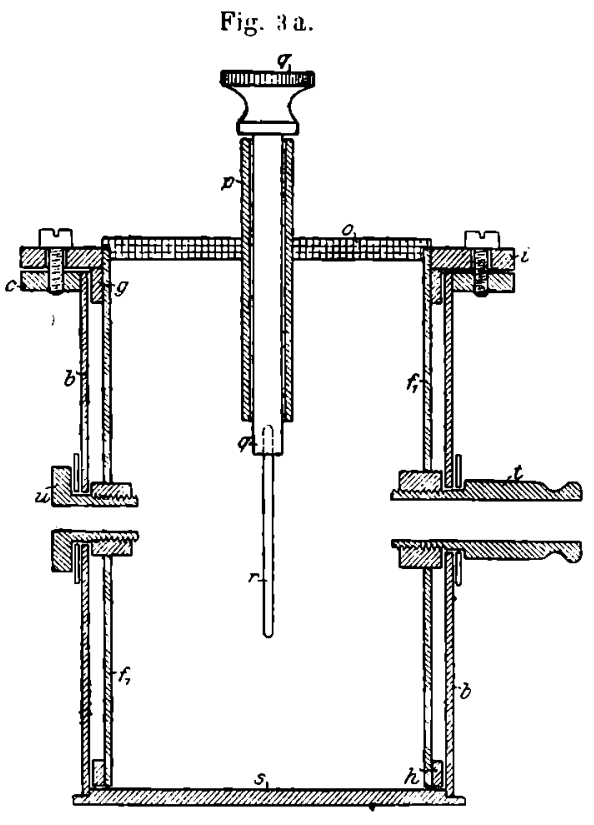
$4 \mathrm{~mm}$ innerem Durchmesser ersetzt (Fig. 3 a), welche man durch dic Durchlochung des äußeren Zylinders $b$ in die des inneren Mantels $f_{1}$ hineinschrauben kann. Hier legt man zur Abdichtung nur Gummiringe zwischen lic Anschlïge der Ansatzröhren $t$ und $n$ und den äußeren Zylinder $b$. Aus dem inneren Messingzylinder $f_{1}$ sind Messingstreifen von $40 \mathrm{~mm}$ Höhe symmelrisclı zu den Durchbohrungen ausgesägt, so daß der obere Teil des Zylinders mit dem unteren nur durch zwei Blechstreifen von $20 \mathrm{~mm}$ Breite an dcr breiteren Durchführung $t$ und von $10 \mathrm{~mm}$ Breite an der engeren Durchführung $u$ in Verbindung steht. Die Blechstreifon überzjeht man innen mit Blei, dn dicses die Röntgenstrahlen außer-

Fig. 3b.

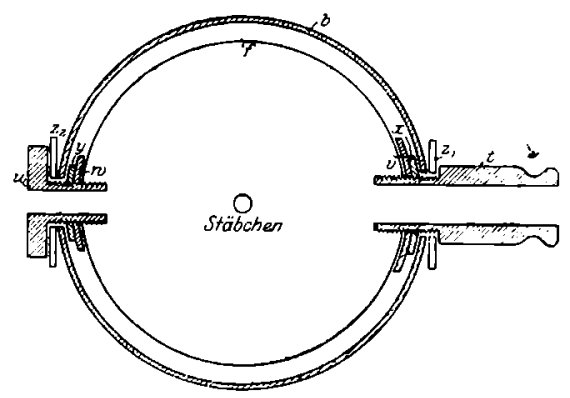
ordentlich wenig reflektiert. Der inncre Zylinder $f_{1}$ wird dann innen mit schwarzem Papier beklebt. Fig. 3b gibt cinen Querschnitt der Kamera in der Höhe der Mitte der Durchführungsröhren $t$ und $u$ wieder. $b$ ist der äußere Zy- 
linder, $v$ und $w$ sind die Streifen des inneren Zylinders $f_{1}$, an welchem die Multern $x$ und $y$ festgelölet sind, in die man die Ansatzröhren $t$ und $u$ hineinschrauben kann. $z^{1}$ und $z^{2}$ sind die Gummiringe zum Abdichten, und $f$ ist der innen am Messingzylinder $f_{1}$ festgeklebte Papierzylinder. Die übrigen Teile sind genau so gebaut wie die der Kamera I. Durch das geringe Volum der Kamera II ist es möglich, nahe an die Antikalhode der Röbre heranzukommen und gleichzeitig drei Kameras an der Antikathodenröhre anzubringen. Mit beiden Typen von Kameras wurden Blindversuche angestellt. Diese ergaben bei 2-3 stündiger Belichtung unmerkliche Reflexe. Bei längerer Belichtung treten sie stärker auf. Sie sind etwas breiter als die von dem Kristallpulver herrührenden, unterscheiden sich aber von diesen in der Form nicht. Sic treten nur in der Nähe der Austrittsstclle des Primärstrahls auf und sind bei 6 stündiger Belichtung etwa so intensiv wic die durch die Reflexion der Kupfer- $\beta$-Strahlung am Kristallpulver erzeugten Linien. Sie dürfen natürlich bei der Auswertung des Films nicht mit benutzt werden.

\section{2. c) Die Blende.}

Um die Röntgenstrahlen auf ein möglichst dünnes Bündel paralleler Strahlen einzuengen, wurde ein Messingröhrchen $a$ (Fig. 4) von $6 \mathrm{~mm}$

Fig. 4.

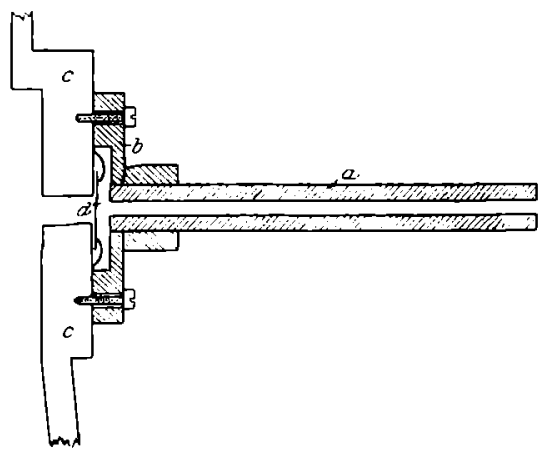
äußerem Durchmesser, welches mit Blei ausgegossen und dann zentrisch zur Röhre durchbohrt wurde, benutzt. Nach der Rüntgenröhre $c$ hin endigt das Röhrchen in einer Messingplatte $b$, durch " welche es senkrecht hindurchführt und an welchem es festgelötet ist. Diese Messingplatte kann an der Antikathodenröhre $c$ so aufgeschraubt werden, daß die Durchbohrung der Blende senkrecht vor dem Aluminiumfenster $d$ steht. Das Rohr $a$ ist bei der Kamera I $70 \mathrm{~mm}$, bei der Kamera Il $50 \mathrm{~mm}$ lang. Es kann durch das weitere Ansatzrohr ( $h$ in Fig. $2, t$ in Fig. 3 a und 3b) der Kamera hindurchgeschoben werden. Bei den Versuchen soll das Ende des Blendenrohres a etwa $4 \mathrm{~mm}$ von der Achse der Kamera entfernt sein.

Soll eine Aufnahme mit möglichst langwelliger Strahlung gemacht werden, so ist es von großem Vorteil, die Kamera zu evakuieren. $\mathrm{Zu}$ diescm Zweck ist Kamera I eingerichtet. Als inneren Zylinder benutzt man dann den der Kamera II ohne Papierbeklebung. Die Ansatzröhre und die Blende werden bei $k$ luftdicht verkittet und üher die Schliffläche bei $c$ 
(Fig. 2) eine Halbkugel von Messing, die unten plangeschliffen ist, aufgeselzt. Das Aluminiumfenster an der Antikathodenröhre wird entfernt und nach den Untersuchungen im Lunder Phys. Inst. durch eine mit Erythrosin gefärbten Goldschlägerhaut ersetzt. Die Blende wird mit der Kamera angeschraubt und $b$ mit $c$ (Fig. 4) verkittet. Die Kamera wird dann durch das Ansatzrohr $l$ evakuiert.

\section{2. d) Das Einlegen der Films.}

Für die Aufnahmen wurden Agfa-Rollfilme $6 \times 9 \mathrm{~cm}$ benutzt. Sie legen sich sehr gut an die äußere Wand des inneren Zylinders der Kamera an. Zum Zurechtschneiden und Durchbohren der Filme benutzt man am besten eine Schablone, welche aus zwei Pappstreifen von $60 \times 155 \mathrm{~mm}$ besteht, dic auf eincr Längsseite durch einen aufgeklebten Leinwandsireifen zusammengehalten werden und in ihrer Mitte eine Durchbohrung von $8 \mathrm{~mm}$ Durchmesser und in der Mitte der Schmalseiten Einschnitte haben. Der Film wird zwischen dic Pappstückc gelegt, in der Länge der Schablone abgeschnitten und mit cinem passenden Korkbohrer an den durch die Schablone angegebenen Stellen durchbohrt. Der zurechtgeschnittene Film wird dann mit der Schichtseite nach innen so auf den äußeren Mantel des inneren Zylinders ( $f$ in Fig. 2 und $f_{1}$ in Fig. 3 a) gelegt, da $B$ dic Durchbohrung des Films über die engere Durchführung des Zylinders, der Austrittsstelle des Primärstrahls, paßt. Über den Film wird dann ein Papierstreifen von $60 \mathrm{~mm}$ Breite gewickelt, der an den Durchführungsstellen der Zylinder der Kamera Löcher besitzt. Film und Papierstreifen werden durch Gummibänder an den inneren Zylinder fest angedrückt. Nun wird der innere Zylinder so in den äußeren geschoben, daß die sich entsprechenden Durchbohrungen beider Zylinder übercinanderfallen. Dann werden die Durchführungsrohre an die Zylinder festgeschraubt und dic Schraube bei $i$ (Fig. 2 und 3 a) angezogen. Man kann nun die Kamera ans Tageslicht bringen, dort das Substanzstäbchen einsetzen und zentrieren.

\section{Das Substanzstäbchen und seine Finstellung.}

Dìe Substanzstälschen künnen in der verschiedensten Art hergestellt werden. Man füllt entweder die gepulverte Substanz in eine Papierhülse, oder man preßt das P'ulver in Stähchenform und überzieht es, wenn dics nötig ist, mit Kollodium, oder man heftet das Pulver an einem Glasstäbchen fest. Solange cine Substanz Papier nicht chemisch verändert, Wasser oder Kohlensäure aus der Luft aufnimmt, ist die Methode der Papierhülse zu bevorzugen; denn es ist gar nicht so empfehlenswert, das Pulver stark zusammenzudrücken, da das Gitter dadurch deformiert werden kann. Außerdem ist bei stark gepreßten Stäbchen die Absorption sehr stark. 
Nimmt eine Substanz Wasser oder Kohlensäure aus der Luft auf, ohne auf das Papier einzuwirken, so überzicht man die Papierhülse nach dem Stopfen des Stäbchens noch mit Paraffin. Dünnes Zigarettenpapier ergab keine Rellexionen, Paraffin nur die bekannten, breiten Reflexionsmaxima, welche nicht mit Kristallreflexionen zu verwechseln sind. Greift eine Sulsstanz Papier an, so überzieht man ein müglichst wenig entglastes, dünnes Glasrührchen mit dem Pulver. Man muß denn von Fall zu Fall prüfen, welche Reflexionen das Glasrührchen für sich allein ergibt. Für den Fall, daß die Pulverpartikelchen durch schwachen Druck so verfestigt werden könden, daß sie ein stabiles Stäbchen ergeben, würde für den letzten Fall diese Methode vorzuziehen scin. Oft kann man sich auch so behelfen, daß man eine Emulsion aus Substanz und Paraffin herstcllt und aus dieser ein Stäbchen formt. Das fertige Pulverstäbchen wird an der drehbaren Achse $q$ (Fig. 2 und 3 a) mit Klebwachs hefestigt und durch die in die Kamera geschobene Blende (Fig. 4) mit einem F'ernrohr beobachtet. Schleudert das Stäbchen beim Drehen der Achse $q$, so nimmt man den Deckel $o$ (Fig. 2 und $3 \mathrm{a}$ ) ab und zentriert solange mit der Hand, bis das Stäbchen beim Drehen der Achse in der Mitte der Blende stehen bleibt. Ist dies erreicht, so schraubt man die Blende mit der Kamera an der Antikathodenröhre vor dem Aluminiumfenster fest.

Sollen drei Aufnahmen glcichzcitig gemacht werden, so setzt man während der Belichtung einen Fluoreszenzschirm hinter eine Kamera. Man kann dann sowohl am Fluoreszenzschirm wie am Milliampèremeter prüfen, ob die Röbre gleichmäßig arbeitet. Werden nur zwei oder nur eine Aufnahme gemacht, so setzt man den Schirm direkt vor ein Fenster der Antikathode.

\section{Die Versuchsbedingungen.}

\section{Größe und Gestalt der Blende und Dicke der Stäbchen.}

Die Blende hat die Aufgahe, ein Bündel möglichst paralleler Strahlen zu erzeugen. Es genügt eine Blende von $50 \mathrm{~mm}$ Länge vollständig, vorausgesetzt, daß sie in der ganzen Länge die gleiche Durchloohrung hat. Die Streuung, die dann noch auftritt, muß in der Rechnung berücksichtigt werden (siehe später Korrektion II). Sie hängt von der Blendenlänge und ferner von der Öffnung der Blende und von dem Stäbchendurchmesser ab. Dieser bedingt außerdem noch eine weitere Korrektion (siehe später Korrektion I). Man wähle, um gute Aufnahmen zu erhalten, den Durchmesser der Blendenöffnung stets $0,2-0,5 \mathrm{~mm}$ grüßer als die Stäbchendicke, so $\mathrm{da} B$ man also sicher ist, $\mathrm{da} B$ die Strahlen den Rand des Stäbchens treffen; denn wir haben es hier mit einem Oberflächeneffekt zu tun $\left.{ }^{1}\right)$.

1) Sielıe später unter Anmerkung zu II, 2. 
Dieses ergab sich unter anderem aus folgendem Versuche: An der Achse der Kamera wurde an Stelle eines Stäbchens ein Pulverblättchen von $0,5 \mathrm{~mm}$ Dicke und etwa $5 \mathrm{~mm}^{2}$ Größe aus Magnesiumoxyd angebracht. Das Primärstrahlenbündel fiel auf dieses senkrechıt auf. Die Aufnahme (Tafel, Film I) zeigt, daß um die Austrittsstelle des Primärstrahlenbündels nur Interferenzlinien von sehr geringer Intensität entstanden sind, während nuf derjenigen Seite des Films, welche an der Eintrittsstelle der Strahlen liegt, sehr scharfe Linien vorhanden sind. Eine Schicht von $0,5 \mathrm{~mm}$ Magnesiumoxydpulver hat also genügt, um die Strahlen so zu absorbieren und zu zerstreuen, daß bei zweistïndiger Belichtung ausmeßbare Linien auf dem Film nicht entstehen künnen. Den Blendendurchmesser wählt man ferner auch deshalb größer als den des Stäbchens, weil dann das Stäbchen besser justiert werden kann.

Für die Ausmessung des Films ist es nötig, intensive Linien von geringer Breite und scharfer Abgrenzung zu erhalten. Es ist lzlar, daß man durch Vergrößerung der refiektierenden Fläche, d. h. durch Vergrößerung von Stäbchendurchmesser und Blendendurchmesser, die Intensiliat der Linien steigern kann. Man erreichl aber dadurch gleichzeitig eine Verbreiterung der Linien. Solange man demnach Blenden mit kreisförmiger ölfnung anwendet, erhült man bei großem Stäbscluen- und Blendendurchmesser intensive und breite Linien auf dem Film und bei kleinem Stäbchen- und Blendendurchmesser wenig intensive und schmale Linien bei gleicher Belichtungszeit. Es wurden daher Versuche mit Spaltblenden angestellt. Das Resultat dieser Versuche zeigt der Film II (Tafel), der mit einer Spaltblende von $4 \mathrm{~mm}$ Länge und 1,5 mm 13reite von einem $1 \mathrm{~mm}$ dicken Magnesiumoxydpulverstäbchen hergestellt wurde. Die Interferenzlinien sind auf diesem lilm in der Mitte schmal, scharf abgegrenzt und intensiver als die, welche von einem Stäbchen von gleichem Durchmesser mit einer kreisförmigen Blende von $2,3 \mathrm{~mm}$ Durchmesser bei gleichen Versuchslıedingungen erhalten wurden. Die re-

DektierendeFläche Fig. $\mathbf{s}$. ist eben bei Verwendung des Spaltes größer. Nach außen zu werden die Linien des Films diffus. Diese Lrscheinung ist so

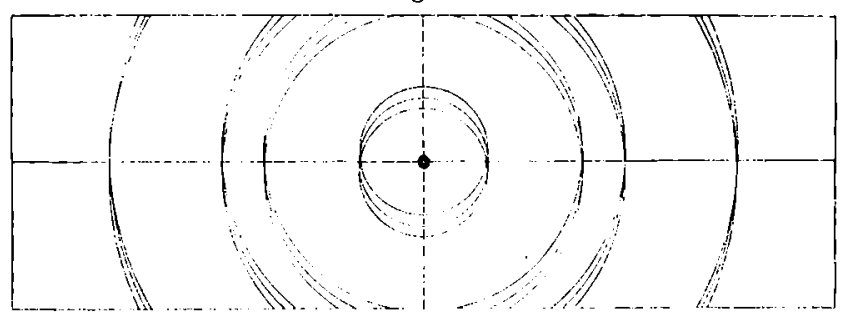
zu erklären: Es entsteht durch dic Vergrößerung der refleklierenden Fläche eine größere Anzahl von Inlerferenzkegeln, die sich auf dem Film überlagern und in der Mitte durchschneiden (Fig. 5; zur Einfachheit sind hier die Interferenzkurven kreisförmig gezeichnel). 
Da allein die Mitte der Linien auf dem Photogramm für die Ausmessung in Frage kommt, fällt ihre Verbreiterung nach dem Rande des Iilms gar nicht ins Gewicht. Sie erleichtert sogar das Auffinden der die Linien durchschneidenden Symmetrieachse, auf welcher die Ausmessung der Abstände der Linien erfolgt. Für die meisten Aufnahmen wurde ein Stäbchen von 1 oder $1,3 \mathrm{~mm}$ Durchmesser und eine Spalthlende von $4 \times 1,5$ oder $4 \times 2 \mathrm{~mm}$ benutzt.

\section{Die Belichtungezeit.}

Schon von rein ökonomischen Gesichtspunkten aus ist es wünschenswert, in möglichst kurzer Zeit einen guten Film zu erhalten. Ferner treten bei längerer Belichtungszeit störende Nebenreflexe stärker hervor als bei kürzerer. Stellt man z. B. von gewissen Substanzen Debye-ScherrerPhotogramme her, so beobachtet man auf dem Film zu beiden Seiten der Eintrittsslelle des Primärstrahls kontinuierliche Schwärzung, welche die Linien um so mehr überdeckt, je länger man belichtet (Tafel, Film III, Anomit von Mliask). Die Schwärzung rührt wahrscheinlich teilweise von einer durch die primäre Strahlung erregten langwelligen Eigenstrahlung her. J)iese Tatsache und die störenden Nehenreflexe, welche durch die Teile der Kamera entstehen können, sprechen ebenfalls für die Verwendung einer Spalthlende, die ja die Bclichtungszeit wesentlich alıkürt.

\section{Die Korngröße des Pulvers.}

Bei sehr geringer Korngröße der Substanzen erhält man Linien von gleichmäßiger Schwärzung. Benutzt man gröberes Pulver zur Aufnahme, so treten oft Scharen von Flecken auf dem Film auf, die alle innerhalb der Interferenzlinie liegen. Diese wurden schon von Debye und Scherrer beobachtet. Nach ihrer Meinung entstehen sie durch Reflexion der Röntgenstrahlen an größeren Kristallpartikelehen im Pulver. Benutzt man für eine solche Aufnahmc eine müglichst enge Spaltblende (Tafel, Film II), so erhält man Striche innerhalb der Interferenzlinie. Diese treten oft scharf abgegrenzt hervor, selbst wenn der Film flau ist. Jhr Vorhandensein deutet oft erst auf Linien hin, die nur bei günstigster Beleuchtung sichtbar werden und daher schwer auszumessen sind. Diese Striche sind daher für die Ausmessung der Filme sehr wertvoll. Beim Ausphotometrieren der Filme wirken sie nicht sonderlich störend. Es ist daher empfehlenswert, die Substanzen nicht zu fein zu pulvern, damit diese Striche entstehen.

\section{Die Auswertang der Films.}

\section{Die Ausmessung der Films.}

Steht ein Apparat zur Ausmessung der Films nicht zur Verfügung, so lotut man sich aus Holz ein kleines Gestell, welches aus einem Grundbrett 
von etwa $140 \times 60 \mathrm{~mm}$ Größe besteht, an dessen beiden Schmalseiten je eine Leiste vertikal befestigt wird. Diese Leisten sind oben so abgeschrägt, daB eine Glasplatte, die auf ihnen befe:tigt wird, unter einem kleinen Winkel vor dem Beschauer liegt. Unter der Glasplatte zwischen den beiden Vertikalleisten befindet sich eine diffus reflektierende Platte (weißes Papier), die zur Variation der Beleuchtungsstärke um ihre rechts-links verlaufende Längsachse drehbar ist. Auf der Glasplatte befesligt man den Film, die Schichtseite der Glasplatte zugewandt. Das diflus reflektierte Licht fällt auf den Film. Durch Drehen der Platte um die Achse kann man die Beleuchlung so regulieren, daß alle Linien des Films sichtbar werden. Über dem Film befestigt man einen möglichst exakt eingeteilten Maßstab. Die Kante desselben muß mit der „Symmelrieachse " des Films zusammenfallen, das heißt, sie muß durch die Milte der Durchstichsstelle des primären Strahlenbündels gehen und die Interferenzlinien senkrecht durchschneiden. Diese Lage kann man durch Drehen des Lineals finden. Exakter ist folgende Melhode: Man legt über den Film einen Streifen Gelatine oder Zelluloid von der Grüße des Films. über diesen Streifen zieht man drei parallele Linien, von denen die zwei äußeren gleiche Entfernung von der mitlleren haben. Durch Drehen des Streifens bringt man die mittlere der Paralleleu in die Richtung der , Symmetrienchse * des Films. Mit einer feinen Spitze sticht man dann die Entfernung der Linien des Films auf den Parallelen ab und mißt die Entfernung der sich entsprechenden Durchstichspunkte zu beiden Seiten der Austriltsstelle des primären Strahlenbündels. Haben die sich auf den äußeren Parallelen entsprechenden Durchstichspunkte beiderseits dieselben Entfernungen voneinander, so geben die Entfernungen auf der mittleren Parallelen die zur Berechnung des Glanzwinkels nötigen Werte an. Sind die Linien eines Films schmaler als $\frac{1}{2} \mathrm{~mm}$, so genügt es, wenn man die Mitte der Linie als Durchslichspunkl wählt. Bei breiteren Linien sticht man an den Rändern jeder Linie des Films auf der miltleren Parallelen des Streifens ein. Die Versuche ergaben bei dreimaligem Messen nach beiden Melhoden stets gute Überciustimmung der Abstandswerte (Fehlergröße $\left.=\frac{1}{10} \mathrm{~mm}\right)$. Aus den Abständen zweier Linien 2 a (Fig. 6) 1) soll der Glanzwinkel $\frac{\delta}{2}$, d. h. der Winkel zwischen dem an einer Kristallfläche reflektierlen Strahl und der Kristallfäche berechnet

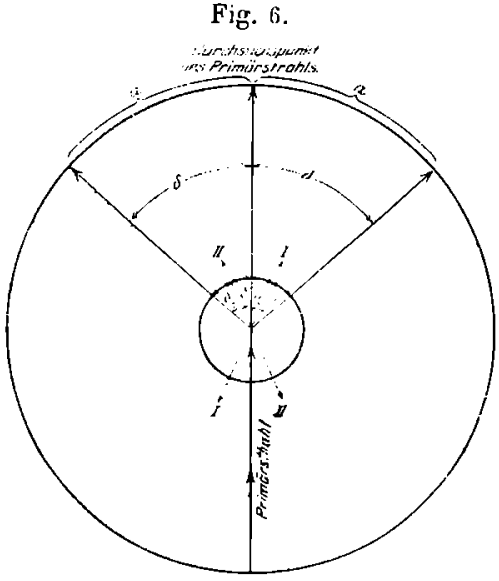

1) In Fig. 6 ist Reflexion der Strahlen im Stähchenmiltelpunkt angenommen. 
werden. Die Entfernung 2a gibt aber den Winkel zwischen den Strahlen an, welche an Flächen (I und II, Fig. 6) reflektiert sind, die zum Primărstrahl unter gleichem Winkel liegen. Da die reflektierende Ebene des Kristalls den Winkel zwischen Primärstrahl und dem an der Fläche reflektierten Strahl halbiert, kommt demnach für die Berechnung des Glanzwinkels der Abstandswert $\frac{a}{2}$ in Betracht.

\section{Die Korrektion auf die Stäbchendicke.}

Da die Reflexion nicht an der (mit der Achse des Films zusammenfallenden) Stäbchenachse erfolgt, sondern an der oberfläche des Stäbchens, ist der aus dem Film gemessene $A$ blenkungswinkel um einen von diesem abhängigen Betrag zu korrigieren $\%$. Zu dessen Berechnung wird angenommen, daß Reflexion praklisch nur in der Oberflächenschicht des Pulverstäbchens erfolgt. Die Berechtigung dieser Annahme ergibt sich weiter aus der Linienbreite, welche der im folgenden entwickelten Ahhängigkeit vom Ablenkungswinkel folgt.

Die Berechnung der Korrektion auf die Stäbchendicke ergibt sich aús Fig. 7. Von dem auf das Stähchen auffallenden Strahlenhündel trägt (vor-

Fig. 7.

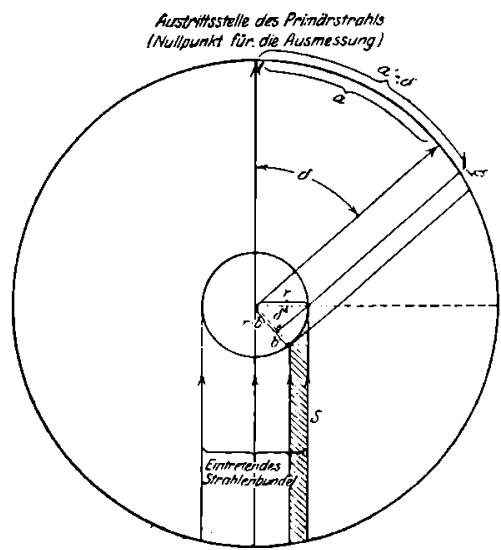
läufig unter der Annahme streng parallelen Rüntgenlichtes) nur der schraffierte Anteil $S$ bei. Er gibt in der Entfernung $a^{\prime}$ von der Austrittsstelle des Primärstrahls eine Reflexionslinie von der Breite $b$. Für die Auswertung benötigt man nicht den $a^{\prime}$ entsprechenden Winkel $\delta^{\prime}$, sondern den winkel $\delta$. Um aus $a^{\prime}$ den Winkel $\delta$ zu berechnen, benutzt man (Fig. 7 ) das recht winklige Dreick mit der Hypothenuse $r$ (Stäbchenradius), der einen Kathele $r-b$ und den von heiden eingeschlossenen Winkel $\delta$ (doppelter Glanzwinkel). Dann ist:

und

$$
r-b=r \cdot \cos \delta
$$

$$
b=r \cdot(1-\cos \delta) \text {. }
$$

In dieser Formel ist also die Linienlreite als Funktion des doppelten Glanzwinkels dargestellt.

1) Die unler II, 2 und II, 3 abgeleiteten Korrektionen gelten streng nur für stark absorbierende Substenzen. Bei sehr schwach absorbierenden Körpern reflektiert niclit nur die Oherflächenschicht des Stäbchens. 
Die Korrektion zur Überführung des gemessenen $a^{\prime}$-Wertes (Entfernung des Schwerpunktes der Linie mit der Breite $b$ bis zur Austrittsstelle des Primärstrahls, welche als Nullpunkt für die Ausmessung angenommen wurde) in die auf Reflexion im Zentrum des Stäbchens bezogene Entfernung $a$ ist unter Benulzung der obigen Formel für $b$ :

$$
r-{ }_{2}^{b}=r-{ }_{2}^{r}+{ }_{2}^{r} \cdot \cos \delta
$$$$
\text { Korrektion }=\frac{r}{2} \cdot(1+\cos \delta) .
$$

Diese Korrektionsgröße ist von $a^{\prime}$ abzuziehen:

$$
a(\text { korrigier } \mathbf{l})=a^{\prime}(\text { gemessen })-\frac{r}{2} \cdot(1+\cos \delta) .
$$

Diesc Kórrektionsformel verlangt die Kenntnis von $\delta$. Da aber $\delta^{\prime}$ von $\delta$ nur um cinen geringen Betrag abweicht, so genügt es, das $\delta$ der Korrektionsformel zu ersetzlen durch:

$$
\delta^{\prime}=\frac{360 \cdot a^{\prime}}{2 \cdot \pi R} .
$$

Jus dem korrigierten $a$ ergibt sich dann der doppelte Glanzwinkel:

$$
\delta=\frac{360 \cdot a}{2 \pi R},
$$

wo $R$ der Radius des Filmzylinders ist.

Die beifolgende Fig. 8 gibt die Korrektionsgröße für Stäbchendurchmesser von $0,5-2 \mathrm{~mm}$ Durchmesser für den gesamten Winkelbereich von

lig. 8.

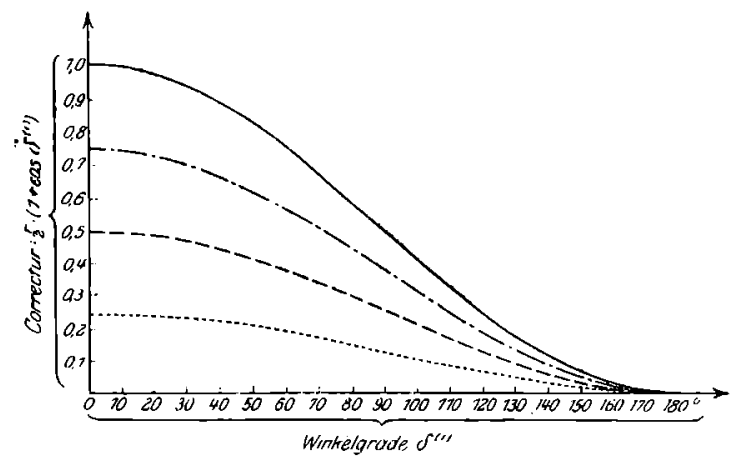

$0^{\circ}-180^{\circ}$ wieder. Diese Korrektionswerte in der Tabelle sind unabhăngig vom Kameraradius. 


\section{Die Korrektion auf die Streuung.}

Die zweite bei der Berechnung des wirklichen Glanzwinkels in Betracht kommende Korrektion ist durch die Streuung bedingt, d. h. durch die Tatsache, daß die Röntgenstrahlen nicht parallel auf das Stäbchen auffallen. Es wird hierbei vorausgesetzt, daß der von dem Stäbchen aus durch die Blende hindurch gesehene Teil der Antikalhode gleichmäßig emittiert. Dann fallen außer parallelen Röntgenstrahlen noch solche auf das Stäbchen, welche mit der Verbindungslinic Stäbchenmitte - Mittelpunkt der Antikathode einen Winkel $\frac{\varphi}{2}$ bilden. Der Winkel $\varphi$ ist gegeben durch die Grenzlinien Stäbchenrand rechts resp. links his innersten Blendenrand links resp. rechts. (Fig. 9.)

Fig. 9.

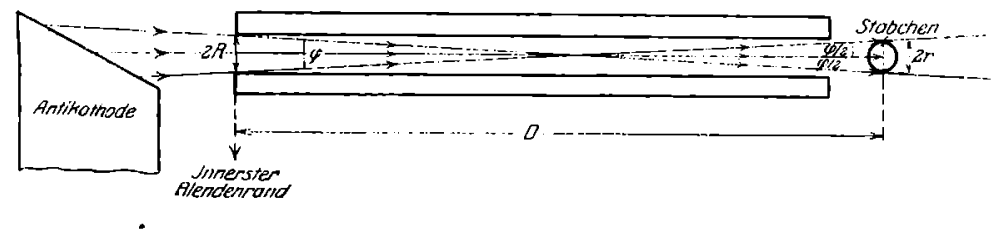

Hierbei ist natürlich vorausgesetzt, daß der Stäbchendurchmesser höchstens die Größe des Austrittsspalts hat idies geschah auch aus anderen Gründen, die unter II. 1. erörtert wurden). Nennen wir den Durchmesser des Stäbchens $2 r$, den Durchmesser des Eintriltsspalts $2 R$ und den Abstand vom Stäbchen bis zum Blendenanfang $D$, so berechnet sich der Streuwinkel aus den ähnlichen Dreiecken (Fig. 9) zu:

$$
\operatorname{tg} \frac{\varphi}{2}=\frac{R+r}{D} \text {. }
$$

Diese Streuung der primären Strahlen hewirkt eine Verschiebung der Reflexionslinie nach grüßeren Winkeln, wie immer von der Austrittsstelle des Primärstrahls aus gerechnet. Die Größe dieser Verschiebung ist streng genommen eine Funktion dieses Winkels. Es genügt jedoch, wie sich durch eine Überschlagsrechnung zeigen läßt, sie konstant zu $\frac{\varphi}{4}$ anzunehmen, d. h. von dem berechneten doppelten Glanzwinkel $\frac{\varphi}{4}$ abzuziehen.

Zur Prüfung beider Korrektionen wurden vom Magnesiumoxyd drei Aufnahmen gemacht, bei welchen der Stäbchendurchmesser zwischen 2,0 und $0,5 \mathrm{~mm}$ variierte. Es ergaben sich dabei innerhalb der Meßgenauigkeit für die Gitlerkonstante folgende Werte: 
Stäbchen-

$\begin{array}{cc}\text { durchmesser } & \text { Spaltbreite } \\ \text { in } \mathbf{m m} & \text { in } \mathbf{m m}\end{array}$

$2 \quad 2,3$

$1 \quad 1,3$

$0,5 \quad 1,3$
Korrektur I in $\mathrm{mm}$

$0,1-0,9$
$0,55-0,44$
$0,4-0,29$

Kantenlänge des

Korrektur II

Elementarwürfels in $\mathrm{cm}$

$$
\begin{aligned}
& 4,219 \cdot 10^{-8} \\
& 4,200 \cdot 10^{-8} \\
& 4,195 \cdot 10^{-8}
\end{aligned}
$$

Trotz sehr verschieden großer Korrektionen erhält man also annähernd gleiche Werte der Gitterkonstanten, ein Beweis für die Hichligkeit der Korrektionen.

\section{Das Ausphotometrieren der Films.}

Von dem Ausphotometrieren der Filme scheinen solange keine quantitativen Resultate zu erwarten zu sein, solange nicht dic Natur des * heflexionsvermögens * aufgeklärt ist, besonders da W. Gerlach und der Verfasser mit den bisherigen Annahmen (Strukturfaktor, Anzahl der reflektierenden Ebenen, Dcly ye-Faktor usw.) nicht erklärbare Intensitätsinomalien gefunden haben. Da wir die Frage noch weiter besonders im Hinblick auf den Zusammenhang mit der Größe und Ladung der Ionen sowie der Packungsdichte verfolgen, soll diese Notiz hier genügen. Zur Strukturaufklärung genügte bisher bei den von uns hergestellten Filmen die Schätzung mit dem Auge.

Hinsichllich der Berechnung der Strukturen aus den Photogrammen sei auf die Arbeiten von Debye und Scherrer ${ }^{1}$, Johnsen und Töplitz $\left.{ }^{2}\right)$ und Runge ${ }^{3}$ ) hingewiesen. Es sei hier nur noch kurz ein Vorschlag von E. Madclung für die Auswertung der Photogramme regulärer Gitter erwähnt.

\section{Graphische Bestimmung der Indizes und der Gitterkonstanten für reguläre Gitter.}

Eine erste graphische Darstellung ergibt den Zusammenhang zwischen dem Linienabstand $m$ und dem zugehörigen Winkel $\frac{360 m}{2 \pi R}$, wo $R$ der

1) a) P. Debye und P. Scherrer, Interferenzen an regellos orientierten Teilchen im Róntgenlicht.

I. Phys. Zeitschr. 1916, 17.

II. Nachr. d. kgl. Ges. d. W. Gottingen vom 18. Dez, 191 3.

III. Phys. Zeitschr. 1917, 18.

b) P. Debye und P. Scherrer, Atombau. Phys. Zeitschr. 1918, 19.

2) A. Johnsen und 0. Toplitz, Über d. math. Auswertung d. Debye-ScherrerRöntgenspektogramme. Phys. Zeitschr. 1918, 19.

3) C. Runge, Die Bestimmung eines Kristallsystems durch Röntgenstrahlen. Phys, Zeitschr. 1917, 18. 
Radius der Kamera ist. Aus dieser Darstellung nimmt man zu den gemessenen $m=a^{\prime}$-Werlen (Fig. 7) die zugehörigen $\left.\delta^{\prime \prime}\right)$-Werte und zu diesen aus einer zweiten graphischen Darstellung $\left(\frac{r}{2} \cdot\left(1+\cos \delta^{\left({ }^{\prime}\right.}\right)\right)$ als Funktion von $\delta^{\prime}$ ) die Korrektionsgröße auf die Stäbchendicke (Fig. 8), um $a^{\prime}$ in $a$ überzuführen. Mittelst der ersten Kurve bestimmt man nun aus diesen $a(m)$-Werten die korrigierten Winkel $\delta$. Von dicsen $\delta$-Werten zieht man die zur jeweiligen Versuchsanordnung gehörige Streuungskorrektion ab und nimmt nun wieder aus der ersten Tabelle die entsprechenden Werte für $a$. Eine dritte graphische Derstellung enthält $\sin ^{2} \frac{\delta}{9}$ als Funktion von $a$ auf der Abszisse und (Fig. 10) auf der Ordinatenachse („Indizesachse «) vom

Fig. 10.

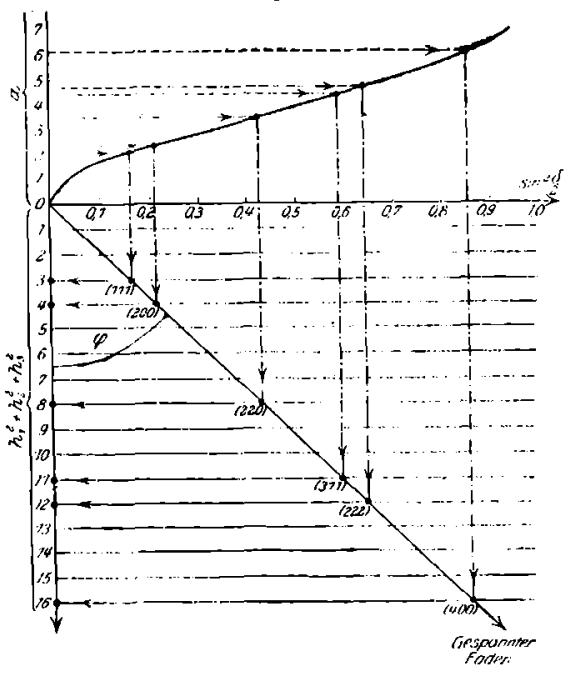

Nullpunkt aus nach unten aufgetragen die Quadratsummen der drei Indizes; durch die so auf der $» I n-$ dizesachse * markierten Punkte sind Parallele zur Abszissenachse gezogen. In dieses letzte Koordinatensystem lotet man die den $a$-Werten des auszuwertenden Films cntsprechenden $\sin ^{2} \frac{\delta}{2}$. Werte. Ein einseitig im Nullpunkt des Koordinatensystems befestigter Faden wird dann (gespannt) so gelegt, daß er durch möglichst zahlreiche Schnittpunkte der genannten $\sin ^{2} \frac{\delta}{2}$-Lote mit den obigen Horizontalen hindurchgeht, die die Indizesquadratsummen darstellen. Hierdurch wird jedem $\sin ^{2} \frac{\delta}{2}$-Wert und somit auch jeder gemessenen Interferenzlinic die richtige Indizesquadratsumme zugeordnet. Nennt man den Neigungswinkel des Fadens gegen die Indizesachse $\varphi$, so ergibt sich:

$$
\operatorname{tg} \varphi=\frac{\sin ^{2} \frac{\delta}{2}}{h_{1}{ }^{2}+h_{2}^{2}+h_{3}^{2}}
$$

wo $h_{1}, h_{2}, h_{3}$ die Indizes sind.

Nun gilt allgemein für reguläre Kristalle, wenn die benulzte Wellenlänge $\lambda$ und $a$ die Kantenlänge des Elementarkuhus bedeutet: 
Aus (1) und (2) folgt:

$$
2 a=\frac{\lambda \cdot \sqrt{h_{1}{ }^{2}+h_{2}^{2}+h_{3}^{2}}}{\sin \delta} .
$$

$$
2 a=\frac{\lambda}{\sqrt{\operatorname{tg} \varphi}} .
$$

Somit ergibt sich aus (3) die Berechnung der Gitterkonslante $a$; zugleich ist klar, daß man für $\lambda_{\alpha}$ und $\lambda_{\beta}$ der Kupferantikathode zweierlei Neigungen $\varphi$ des Fadens erhält.

Der experimentelle Teil der Arbeit wurde im Institut für theoretische Physik der Universität Frankfurt ausgeführt. Dem früheren Direktor des Instituts, Herrn M. Born und seinem Nachfolger, Herrn E. Madelung, bin ich für die liebenswürdige Art, in der sie mir die Mittel ihres Instituts zur Verfügung stellten, und für das lebhafte Interesse an der Arbeit zu größtem Dank verpflichtet. Die benutzte Transformatoranlage ist Herrn M. von Lauc vom Institut int. de Physik Solvay gestiftet worden. Für die leihweise ÜLerlassung derselben sage ich Hern M. von Laue meinen verbindlichsten Dank. Ebenso möchte ich Herrn W. Gerlach für seine fleißige Mitarbeit an den bisher gemeinschaflich angefertigten Strukturarbeiten, die für die vorliegende Arbeit sehr wertvoll waren, herzlich danken. Dem Institutsmechaniker, Herrn A. Schmidt, müchte ich ferner meinen herzlichsten Dank für die wertvolle Hilfe bei der Herstellung der Apparatur aussprechen.

Frankfurt a. M., Institut für theoretische Physik, Oktober 1921. 